Article

\title{
A Stochastic Poisson Structure
}

\section{Rémi Léandre}

Institut de Mathématiques, Université de Bourgogne, 21000 Dijon, France;

E-Mail:Remi.Leandre@u-bourgogne.fr

Received: 2 July 2009; in revised form: 26 July 2009 / Accepted: 12 August 2009 /

Published: 20 August 2009

\begin{abstract}
We define a Poisson structure on the Nualart-Pardoux test algebra associated to the path space of a finite dimensional Lie algebra.
\end{abstract}

Keywords: Poisson structure; Malliavin Calculus

\section{Introduction}

Deformation quantization in field theory is an interesting subject [1-3]. Motivated by that, Dito ([4]) has considered the canonical Poisson structure on $\mathbb{H} \oplus \mathbb{H}^{*}$ where $\mathbb{H}$ is a Hilbert space. Dito has defined the Moyal product on the algebra of Hilbert-Schmitt smooth functions on $\mathbb{H} \oplus \mathbb{H}^{*}$.

Models of stochastic analysis, if they are similar to models of quantum field theory, are more tractable. As a consequence, infinite dimensional analysis, although very similar to highly studied objects of quantum field theory, is much more easier to handle.

There are more or less two types of infinite dimensional analysis:

-)The Malliavin Calculus, which is analytical.

-)White noise analysis, which is algebraic.

Dito-Léandre [5] has interpreted [4] inside the Malliavin Calculus, the algebra being the Malliavin test algebra of functionals smooth in the Malliavin sense on the Wiener space.

Léandre has developed the paper [5] by considering more regular symplectic structure which leads to the study of more singular Poisson structure in [6-9], by considering the Hida test algebra endowed with a normalized Wick product instead of the Malliavin test algebra endowed with the Wiener product. The main difference between white noise analysis and the Malliavin Calculus is that the Hida test algebra is much more smaller than the Malliavin test algebra. The space of allowed operations in white noise analysis is much more bigger than the space of allowed operations in the Malliavin Calculus. 
For a review of deformation quantization in infinite dimensional analysis, we refer to the review of Léandre [7].

There are a lot of works on Poisson structure in infinite dimension [10], in part motivated by the theory of integrable systems. We refer to the review of Mokhov [11] and Dubrovin-Novikov [12] for that.

We would like to give an analytical meaning to the simplest Poisson structure considered in the review of Dubrovin-Novikov [12] formula (44) p 53). Let us recall how it works. We consider the set of paths $t \rightarrow x(t)$ from $[0,1]$ into $\mathbb{R}^{m}$. The Poisson structure is defined by

$$
\left\{x_{i}(s), x_{j}(t)\right\}=\delta_{0}(s-t) \sum_{k} c_{i, j}^{k} x_{k}(t)
$$

$c_{i, j}^{k}$ are the structural coefficient of a Lie algebra structure on $\left(\mathbb{R}^{m}\right)^{*} ; \delta_{0}$ is the Dirac mass in 0 .

In white noise analysis, people replace the Poisson bracket $\{$,$\} by a Lie bracket [$,$] , in order to get a$ representation of the canonical commutation relations on the Hida Fock space. The white noise $x(t)$ is classically represented as $a(t)+a(t)^{*}$, where

$$
\begin{gathered}
{[a(t), a(s)]=\left[a^{*}(s), a^{*}(t)\right]=0} \\
{\left[a(t), a^{*}(s)\right]=\delta_{0}(s-t)}
\end{gathered}
$$

So we have to identify $x(t)$ as a white noise in (1) and not as a Brownian motion, in order to understand analytically the Poisson structure (1). But this Poisson structure will lead to the introduction of anticipative Stratonovich integrals. The Malliavin test algebra is not adequate to understand anticipative Stratonovich integrals.

Nualart-Pardoux pioneered the study of anticipative Stratonovich integrals in [13]. Functional spaces were suitably defined by Léandre [14-18], in order to understand anticipative Stratonovich integrals in a convenient way.

Léandre has defined a Nualart-Pardoux Calculus, which is a refinement of the Malliavin Calculus and allows to define various stochastic cohomology theories on various loop spaces ([14-18]). The NualartPardoux test algebra N.P $P_{\infty_{-}}$is smaller than the Malliavin test algebra for the Brownian motion on $\mathbb{R}^{m}$. But the anticipative Statonovich integrals act continuously on N.P $P_{\infty_{-}}$. We get the main theorem of this work:

Theorem 1 The Poisson structure $\{$,$\} in (1) acts continuously on N.P P_{\infty-}$

\section{A Brief Review on the Nualart-Pardoux Calculus}

We consider the set of continuous paths $C\left([0,1] ; \mathbb{R}^{m}\right)$ from $[0,1]$ into $\mathbb{R}^{m}$ endowed with the uniform topology. A typical path is denoted by $t \rightarrow B(t)=\left(B_{i}(t)\right)$, on which we consider the Brownian motion measure $d P([19])$.

Let us recall how we construct $d P$. We consider the Cameron-Martin Hilbert space $H$ [20-21] of maps from $[0,1]$ into $\mathbb{R}^{m}$ such that

$$
\int_{0}^{1}\left|h_{s}^{\prime}\right|^{2} d s=\|h\|^{2}<\infty
$$


$d P$ is formally the Gaussian probability measure

$$
1 / Z \exp \left[-\|h\|^{2} / 2\right] d D(h)
$$

$d D$ is the formal Lebesgue measure on $H$ which does not exist as a measure (We refer to the works of Léandre [22-25], Asada [26], Pickrell [27] for various approaches to the Lebesgue measure in infinite dimension). Let $H_{1}$ be a finite dimensional real Hilbert space $\left(h_{1} \in H_{1}\right)$ with Hilbert norm \|\|$_{1}$. Let us consider the centered normalized Gaussian measure on $H_{1}$. It is classically represented by $\sum e_{i} N_{i}$ where $N_{i}$ are centered normalized independent one dimensional Gaussian variables and the system of $e_{i}$ constitutes an orthonormal basis of the real Hilbert space $H_{1}$.

It should be tempting to represent $d P$ by using the same procedure. We consider an orthonormal basis $e_{i}$ of $H$. The law of the Brownian motion is represented by the series $\sum e_{i} N_{i}$ where the $N_{i}$ is a collection of independent centered one dimensional Gaussian variables. This series does not converge in $H$ but in $C\left([0,1] ; \mathbb{R}^{m}\right)$ [28]. We refer to the textbook of Kuo [29] for the theory of infinite dimensional Gaussian measures.

Let us consider a functional $F$ on $C\left([0,1] ; \mathbb{R}^{m}\right)$. Its $r^{\text {th }}$ stochastic derivative $d^{r} F$, according to the framework of the Malliavin Calculus [30-32], is defined if it exists by

$$
d^{r} F\left(h_{1}, . ., h_{r}\right)=\int_{[0,1]^{r}}<d^{r} F\left(s_{1}, . ., s_{r}\right), h_{1, s_{1}}^{\prime}, . . h_{r, s_{r}}^{\prime}>d s_{1} . . d s_{r}
$$

where $h_{i}$ belongs to the Hilbert space of paths $H$ from $[0,1]$ into $\mathbb{R}^{m}$ such that

$$
\int_{0}^{1}\left|h_{s}^{\prime}\right|^{2} d s=\|h\|^{2}<\infty
$$

The Sobolev norms of the Malliavin Calculus are defined by the following formula. If $F$ is a Brownian functional,

$$
E\left[\left(\int_{[0,1]^{r}}\left|d^{r} F\left(s_{1}, . ., s_{r}\right)\right|^{2} d s_{1} . . d s_{r}\right)^{p / 2}\right]^{1 / p}=\|F\|_{r, p}
$$

The Malliavin test algebra is consisted of Brownian functionals $F$ such that for all $r, p,\|F\|_{r, p}<\infty$. Let us recall how we construct these Sobolev spaces. Let $f$ be a smooth function from $\left(\mathbb{R}^{m}\right)^{d}$ into $\mathbb{R}$ with compact support and some times $0<t_{1}<. .<t_{d} \leq 1$. We introduce the cylindrical functional $F(B())=.f\left(B\left(t_{1}\right), . ., B\left(t_{d}\right)\right)$. We consider the Gateaux derivative of $F$ along a deterministic direction $h$ of $H$ :

$$
<d F, h>=<\sum \frac{\partial}{\partial x_{i}} f\left(B\left(t_{1}\right), . . B\left(t_{d}\right)\right), h_{t_{i}}>
$$

There is absolutely no problem to define it. We have the integration by parts formula true for any cylindrical functional

$$
E[<d F, h>]=E\left[F \int_{0}^{1}<h_{s}^{\prime}, \delta B(s)>\right]
$$

where $\delta B(s)$ is the Itô differential. The Itô integral is the limit in all the $L^{p}(d P) p<\infty$ of the sum $\sum<h_{s_{i}}^{\prime}, B\left(s_{i+1}\right)-B\left(s_{i}\right)>$ where $0<s_{1}<. .<s_{i}<s_{i+1}<. .<s_{2^{n}-1}<1=s_{2^{n}}$ is a dyadic subdivision of $[0,1]$ of length $2^{n}$. The convergence does not pose any problem because $h$ is deterministic. Since we have the integration by parts formula (10), we can extend the operation of taking the stochastic 
derivative of a Brownian functional $F$ consistently, as we establish classically the definition of Sobolev spaces in finite dimension. The main novelty of the Malliavin Calculus with respects of its precursors motivated by mathematical physics [33-36] is that the algebra of functionals which belong to all the Sobolev spaces of the Malliavin Calculus (these functionals are said to be smooth in the Malliavin sense) is constituted of functionals almost surely defined. The reader interested in the Malliavin Calculus can see the books $[30,32]$.

If we consider the same dyadic subdivision as before, we can introduce the polygonal approximation $B^{n}(t)$ of $B(t)$. Let us consider a (non deterministic!) map from $[0,1]$ into $\mathbb{R}^{m} t \rightarrow h_{t}$ which belongs to $L^{2}\left([0,1] ; \mathbb{R}^{m}\right)$. We can consider the random ordinary integral $\int_{0}^{1}<h_{t}, d B^{n}(t)>$. It has no problem to be defined. To pass to the limit when $n \rightarrow \infty$, there are a lot of problems. If we can pass to the limit, we say that the limit $\int_{0}^{1}<h_{t}, d B(t)>$ is an anticipative Stratonovich integral. Nualart-Pardoux [13] are the first authors who have defined some anticipative Stratonovich integrals. A convenient theory was established by Léandre [14-18] in order to understand some Sobolev cohomology theories on the loop space. Let us recall it quickly.

We consider another set of Sobolev norms ([14]). We suppose that outside the diagonals of $[0,1]^{r}$

$$
E\left[\left|d^{r} F\left(s_{1}, . ., s_{r}\right)-d^{r} F\left(s_{1}^{\prime}, . ., s_{r}^{\prime}\right)\right|^{p}\right]^{1 / p} \leq C_{r, p} \sum\left|s_{i}-s_{i}^{\prime}\right|^{1 / 2}
$$

The smallest $C_{r, p}$ such that the previous inequality is satisfied is called the first Nualart-Pardoux Sobolev norm. The second Nualart-Pardoux Sobolev norm is the smallest $C_{r, p}^{1}$ such that for all $\left(s_{i}\right) \in$ $[0,1]^{r}$,

$$
E\left[\left|d^{r} F\left(s_{1}, . ., s_{r}\right)\right|^{p}\right]^{1 / p} \leq C_{r, p}^{1}
$$

Definition 2 The Nualart-Pardoux test algebra N.P $P_{\infty_{-}}$is consisted of functionals $F$ whose all NualartPardoux Sobolev norms of first type and second type are finite. Elements $F$ of N.P. $P_{\infty}$ are said to be smooth in the Nualart-Pardoux sense.

Let us recall that $N . P_{\infty_{-}}$is an algebra [14].

We can consider a random element of $L^{2}\left([0,1] ; \mathbb{R}^{m}\right) t \rightarrow h_{t}$. We can consider its $r^{t h}$ stochastic derivative

$$
d^{r} h_{t}\left(h_{1}, . ., h_{r}\right)=\int_{[0,1]^{r}}<d^{r} h_{t}\left(s_{1}, . ., s_{r}\right), h_{1, s_{1}}^{\prime}, . . h_{r, s_{r}}^{\prime}>d s_{1} . . d s_{r}
$$

Its first Nualart-Pardoux Sobolev norm $C_{r, p}$ is the smallest number such that outside the diagonals of $[0,1] \times[0,1]^{r}$

$$
E\left[\left|d^{r} h_{t}\left(s_{1}, . ., s_{r}\right)-d^{r} h_{t^{\prime}}\left(s_{1}^{\prime}, . ., s_{r}^{\prime}\right)\right|^{p}\right]^{1 / p} \leq C_{r, p}\left(\left|t-t^{\prime}\right|^{1 / 2}+\sum\left|s_{i}-s_{i}^{\prime}\right|^{1 / 2}\right)
$$

The second type of Nualart-Pardoux Sobolev norm $C_{r, p}^{1}$ of $h_{(.)}$is the smallest number such that for all $\left(t, s_{1}, . ., s_{r}\right) \in[0,1] \times[0,1]^{r}$

$$
E\left[\left|d^{r} h_{t}\left(s_{1}, . ., s_{r}\right)\right|^{p}\right]^{1 / p} \leq C_{r, p}^{1}
$$

Let us recall the theorem of Léandre [14]: 
Theorem 3 Let $h$ be a random element of $L^{2}\left([0,1] ; \mathbb{R}^{m}\right)$ such that all its Nualart-Pardoux Sobolev norm are finite. Then the anticipative Stratonovich integral

$$
\int_{0}^{1}<h_{t}, d B(t)>
$$

is smooth in the Nualart-Pardoux sense and its Nualart-Pardoux Sobolev norms can be estimated in terms of the Nualart-Pardoux norms of $H$.

In such a case, $\int_{0}^{1}<h_{t}, d B(t)>$ is the limit in all the $L^{p}(d P) p<\infty$ of $\int_{0}^{1}<h_{t}, d B^{n}(t)>$.

Moreover,

$$
<d\left(\int_{0}^{1}<h_{t}, d B(t)>\right), \tilde{h}>=\int_{0}^{1}<<d h_{t}, \tilde{h}>, d B(t)>+\int_{0}^{1}<h_{t}, d / d t \tilde{h}_{t}>d t
$$

This means that the kernel of the stochastic derivative of $\int_{0}^{1}<h_{t}, d B(t)>$ is $\int_{0}^{1}<d h_{t}(s), d B(t)>$ $+h_{s}$.

Let us explain this formula: in order to take the stochastic derivative of $\int_{0}^{1}<h_{t}, d B(t)>$, we do the same formal computations as if the anticipative Stratonovich integral had been a classical integral: we take first of all derivatives of $h_{t}$ which lead to the term $<d h_{t}, \tilde{h}>$ and derivative of $d B_{t}$ which lead to $d / d t \tilde{h}_{t} d t$.

\section{The Poisson Structure on the Nualart-Pardoux Test Algebra}

Let us recall what is a Poisson bracket $\{$,$\} . We consider a commutative Frechet unital real algebra$ endowed with a family of Banach norms $\|.\|_{p}$. This means that for all $p$, there exists $p^{1}$ such that for all $F^{1}, F^{2}$ in $A$

$$
\left\|F^{1} F^{2}\right\|_{p} \leq C_{p}\left\|F^{1}\right\|_{p^{1}}\left\|F^{2}\right\|_{p^{1}}
$$

A Poisson Bracket is a bilinear map from $A \times A$ into $A$, which is a derivation in each argument, vanishes on the unit. The derivation property means that for all $F^{1}, F^{2}, F^{3}$ in $A$

$$
\left\{F^{1} F^{2}, F^{3}\right\}=F^{1}\left\{F^{2}, F^{3}\right\}+\left\{F^{1}, F^{3}\right\} F^{2}
$$

Moreover, it satisfies the following properties: If $F^{1}, F^{2}, F^{3}$ belong to $A$,

$$
\begin{gathered}
\left\{F^{1}, F^{2}\right\}=-\left\{F^{2}, F^{1}\right\} \\
\left\{\left\{F^{1}, F^{2}\right\}, F^{3}\right\}+\left\{\left\{F^{2}, F^{3}\right\}, F^{1}\right\}+\left\{\left\{F^{3}, F^{1}\right\}, F^{2}\right\}=0
\end{gathered}
$$

Moreover, for all $p$, there exists $p^{\prime}$ such that

$$
\left\|\left\{F^{1}, F^{2}\right\}\right\|_{p} \leq C\left\|F^{1}\right\|_{p^{\prime}}\left\|F^{2}\right\|_{p^{\prime}}
$$

In the sequel, we will choose $A=N . P_{\infty_{-}}$. We consider the structural constants $c_{i, j}^{k}$ of a Lie algebra structure on $\left(\mathbb{R}^{m}\right)^{*}$. The stochastic gradient $d F$ of a functional $F$ can be written $d F=\left(d F_{i}\right)$. Formula (1) reads in this framework

$$
\left\{F^{1}, F^{2}\right\}=\sum_{i, j, k} \int_{0}^{1} d F_{i}^{1}(s) d F_{j}^{2}(s) c_{i, j}^{k} d B_{k}(s)
$$


where we consider a Stratonovitch anticipative integral.

Proof of Theorem $1 \mathrm{In}$ order to show that (19) is satisfied, we remark that $d\left(F^{1} F^{2}\right)=F^{1} d F^{2}+$ $F^{2} d F^{1}$. Moreover, since we consider anticipative Stratonovich integrals, we have $\int_{0}^{1}<g h_{t}, d B_{t}>=$ $g \int_{0}^{1}<h_{t}, d B_{t}>$ almost surely. Then (19) is satisfied.

Only the Jacobi relation (21) put a problem. We have:

$$
\begin{aligned}
d_{i^{4}}\left\{F^{1}, F^{2}\right\}(t)= & \sum_{i^{1}, i^{2}, k} \int_{0}^{1} d_{i^{1}, i^{4}} F^{1}(s, t) d_{i^{2}} F^{2}(s) c_{i^{1}, i^{2}}^{k} d B_{k}(s) \\
& +\sum_{i^{1}, i^{2}, k} \int_{0}^{1} d_{i^{2}, i^{4}} F^{2}(s, t) d_{i^{1}} F^{1}(s) c_{i^{1}, i^{2}}^{k} d B_{k}(s)+\sum_{i^{1}, i^{2}} d_{i^{1}} F^{1}(t) d_{i^{2}} F^{2}(t) c_{i^{1}, i^{2}}^{i_{4}}
\end{aligned}
$$

Therefore

$$
\begin{aligned}
& \left\{\left\{F^{1}, F^{2}\right\}, F^{3}\right\}= \\
& \quad \sum_{i^{1}, i^{2}, i^{3}, i^{4}, k, k^{\prime}} \int_{[0,1]^{2}} d_{i^{1}, i^{4}} F^{1}(s, t) d_{i_{2}} F^{2}(s) d_{i^{3}} F^{3}(t) c_{i^{1}, i^{2}}^{k} c_{i^{4}, i^{3}}^{k^{\prime}} d B_{k}(s) d B_{k^{\prime}}(t) \\
& +\sum_{i^{1}, i^{2}, i^{3}, i^{4}, k, k^{\prime}} \int_{[0,1]^{2}} d_{i^{1}} F^{1}(s) d_{i^{2}, i^{4}} F^{2}(s, t) d_{i^{3}} F^{3}(t) c_{i^{1} i^{2}}^{k} c_{i^{4} i^{3}}^{k^{\prime}} d B_{k}(s) d B_{k^{\prime}}(t) \\
& \quad+\sum_{i^{1}, i^{2}, i^{3}, i^{4}, k} \int_{0}^{1} d_{i^{1}} F^{1}(s) d_{i^{2}} F^{2}(s) d_{i^{3}} F^{3}(s) c_{i^{1}, i^{2}}^{i_{4}} c_{i^{4}, i^{3}}^{k} d B_{k}(s)=A_{1,2,3}+B_{1,2,3}
\end{aligned}
$$

If $e_{i}$ is the canonical basis of $\left(\mathbb{R}^{m}\right)^{*}$, we have

$$
\left[e_{i}, e_{j}\right]=\sum c_{i, j}^{k} e_{k}
$$

such that the Jacobi relation

$$
\left[\left[e_{i^{1}}, e_{i^{2}}\right], e_{i^{3}}\right]+\left[\left[e_{i^{2}}, e_{i^{3}}\right], e_{i^{1}}\right]+\left[\left[e_{i^{3}}, e_{i^{1}}\right], e_{i^{2}}\right]=0
$$

reads

$$
\sum_{k^{\prime}} c_{i^{1}, i^{2}}^{k^{\prime}} c_{k^{\prime}, i^{3}}^{k}+\sum_{k^{\prime}} c_{i^{2}, i^{3}}^{k^{\prime}} c_{k^{\prime}, i^{1}}^{k}+\sum_{k^{\prime}} c_{i^{3}, i^{1}}^{k^{\prime}} c_{k^{\prime}, i^{2}}^{k}=0
$$

Therefore,

$$
B_{1,2,3}+B_{2,3,1}+B_{3,1,2}=0
$$

Let us show that

$$
A_{1,2,3}+A_{2,3,1}+A_{3,1,2}=0
$$

The second derivative of $F^{1}$ appears only in $\left\{\left\{F^{1}, F^{2}\right\}, F^{3}\right\}$ and in $\left\{\left\{F^{3}, F^{1}\right\}, F^{2}\right\}$. More precisely $d_{i^{1}{ }^{4}} F(s, t)$ appears in

$$
\begin{aligned}
\sum_{i^{2}, i^{3}, k, k^{\prime}} \int_{[0,1]^{2}} d_{i^{1}, i^{4}} F^{1}(s, t) d_{i^{2}} F^{2}(s) d_{i^{3}} F^{3}(t) c_{i^{1}, i^{2}}^{k} c_{i^{4}, i^{3}}^{k^{\prime}} d B_{k}(s) d B_{k^{\prime}}(t) & \\
& +\sum_{i^{2}, i^{3}, k, k^{\prime}} \int_{[0,1]^{2}} d_{i^{3}} F^{3}(s) d_{i^{1} i^{4}} F^{1}(s, t) d_{i^{2}} F^{2}(t) c_{i^{3}, i^{1}}^{k} c_{i^{4}, i^{2}}^{k^{\prime}} d B_{k}(s) d B_{k^{\prime}}(t)=A_{i^{1}, i^{4}}
\end{aligned}
$$


But by the Schwarz relation

$$
d_{i^{1} i^{4}} F^{1}(s, t)=d_{i^{4} i^{1}} F^{1}(t, s)
$$

Moreover $\left[e_{i}, e_{j}\right]=-\left[e_{j}, e_{i}\right]$ such that

$$
c_{i^{3}, i^{1}}^{k}=-c_{i^{1}, i^{3}}^{k}
$$

This shows that

$$
\sum_{i^{1}, i^{4}} A_{i^{1}, i^{4}}=0
$$

\section{Acknowledgments}

We thank B. Dubrovin and V. Rubtsov for helpful discussions.

\section{References}

1. Dito, G. Star-product approach to quantum field theory: the free scalar field. Let. Math. Phys. 1990, 20, 125-134.

2. Dito, G. Star-products and nonstandard quantization for Klein-Gordon equation. J. Math. Phys. 1992, 33, 791-801.

3. Duetsch, M.; Fredenhagen, K. Perturbative algebraic field theory and deformation quantization. In "Mathematical physics in Mathematics and Physics", Fields Inst. Commun. 30, A.M.S., 2001, 151-160.

4. Dito, G. Deformation quantization on a Hilbert space. In Noncommutative geometry and physics; Maeda, Y., Ed.; World Scientific: Hackensack, NJ, USA, 2005; 139-157.

5. Dito, G.; Léandre, R.: Stochastic Moyal product on the Wiener space. J. Math. Phys. 2007, 48, 023509.

6. Léandre, R. Deformation quantization in white noise analysis. In Geometric aspects of integrable systems; Calogero, F., Françoise, J.P., Marrero, J.C., Manojlovic, N., Nunes da Costa, J., Eds.; S.I.G.M.A. 2007 3, 027 (Electronic).

7. Léandre, R. Deformation quantization in infinite-dimensional analysis. In Trends in Stochastic Analysis (Festchrift in honour of H.v. Weizsaecker), Kaiserslautern, Germany, 2007; Scheutzow, M., Moters, P., Blath, J., Eds.; Cambridge University Press: Cambridge, U.K., 2009; pp. 283-303.

8. Léandre, R. Fedosov quantization in white noise analysis. In NEEDS 2007, Almetla-del-Mar, Spain, 2007; Gomez-Ullate, D., Hone, A., Lombardo, S., Puig i Sadurni, J., Eds.; J. Nonlinear Math. Phys. 2008, 15, Supplement 3, 251-263 (Electronic).

9. Léandre, R. Hochschild cohomology theories in white noise analysis. In Special issue on Deformation Quantization; Cattaneo, A., Dito, G., Kontsevich, M., Sternheimer, D., Eds., S.I.G.M.A. 2008, 4, 066 (Electronic).

10. Odzijewicz, A.; Ratiu, T. Banach Lie-Poisson spaces and reduction. Comm. Math. Phys. 2003, 243, 1-54.

11. Mokhov, O.I. Differential geometry of symplectic and Poisson structures on loop spaces of smooth manifolds and integrable systems. In Loop spaces and groups of diffeomorphisms, Proc. Steklov. Inst. 1997, 217, 91-125. 
12. Dubrovin, B.; Novikov, S. Hydrodynamics of weakly deformed soliton lattices. Differential geometry and Hamiltonian theory. Rus. Math. Survey. 1989, 44, 35-124.

13. Nualart, D.; Pardoux, E. Stochastic calculus with anticipating integrals. Probab. theory. rel. Field. 1988, 78, 535-581.

14. Léandre, R. Cohomologie de Bismut-Nualart-Pardoux et cohomologie de Hochschild entiere. In Séminaire de probabilités XXX en l'honneur de P.A. Meyer et J. Neveu; Azéma, J., Emery, M., Yor, M. Eds. Lecture. Notes. Math 1626, Springer: Heidelberg, Germany, 1996; pp. 68-100.

15. Léandre, R. Brownian cohomology of an homogeneous manifold. In New trends in stochastic analysis, A. Taniguchi Conference, Charingworth, England, 1994; Elworthy, K.D., Kusuoka, S., Shigekawa, I. Eds.; World Scientific: Singapore, Singapore, 1997; pp. 305-347.

16. Léandre, R. Cover of the Brownian Bridge and stochastic symplectic action. Rev. Math. Phys. 2000, 12, 91-137.

17. Léandre, R. Stochastic Adams theorem for a general compact manifold. Rev. Math. Phys. 2001, 13, 1095-1134.

18. Léandre, R. Stochastic algebraic de Rham complexes. Acta. Appli. Mathematicae. 2003, 79, 217-247.

19. Lévy, P. Processus stochastiques et mouvement Brownien, 3rd ed.; Gauthier-Villars: Paris, France, 1992.

20. Cameron, R.H. The first variation of an indefinite Wiener integral. Proc. Amer. Math. Soc. 1951, 2, 914-924.

21. Cameron, R.H.; Martin, W.T. Transformations of Wiener integrals under translations. Ann. of. Maths. 1944, 4, 386-396.

22. Léandre, R. Paths integrals in noncommutative geometry. In Encyclopedia of Mathematical Physics; Françoise, J.P., Naber, G.L., Tsun, T.S., Eds.; Elsevier: Oxford, UK, 2006; pp. 8-12.

23. Léandre, R. Infinite Lebesgue distribution on a current group as an invariant distribution. In Foundations of Probability and Physics IV, Vaxjoe, Sweden, 2006; Adenier, G., Fuchs, C., Khrennikov, A., Eds.; AIP Proceedings 889, A.IP., Melville 2007; pp. 332-336.

24. Léandre, R. Lebesgue measure in infinite dimension as an infinite dimensional distribution. In Conference in honour of Y.P. Solovyov, Moscow, Russia, 2005; Mishchenko, A., Ed.; Fundamental and applied mathematics (Russian/English) 2007, 13, 127-132. J. Math. Sci. 2009, 159, 833-836.

25. Léandre, R. Long time behaviour of the Wiener process on a path group. To appear in Group Theory: Classes, Representations and Connections, and Applications.

26. Asada, A. Regularized Calculus: an application of zeta regularization to infinite dimensional geometry and analysis. Int. J. Geom. Mod. Phys. 2004, 1, 107-157.

27. Pickrell, D. Invariant measures for unitary groups associated to Kac-Moody Lie alegras; Mem. A.M.S. 693, A.M.S.: Providence, USA, 2000.

28. Neveu, J. Processus aléatoires gaussiens; Presses Univ. Montréal: Montréal, Canada, 1968.

29. Kuo, H.H. Gaussian measures in Banach spaces; Lecture. Notes. Math. 463, Springer: Heidelberg, Germany, 1975.

30. Ikeda, N.; Watanabe, S. Stochastic differential equations and diffusion processes, 2nd ed; NorthHolland: Amsterdan, The Netherlands, 1989. 
31. Malliavin, P. Stochastic calculus of variations and hypoelliptic operators. In Proceedings of the International Symposium on Stochastic Differential Equations, Kyoto, Japan, 1976; Itô, K. Ed.; Wiley: New-York, U.S.A., 1978; pp. 195-263.

32. Nualart, D. The Malliavin Calculus and related topics; Springer: Heidelberg, Germany, 1995.

33. Albeverio, S.; Hoegh-Krohn, R. Dirichlet forms and diffusion processes on rigged Hilbert spaces. Z.W. 1977, 40, 1-57.

34. Berezanskii, Y. The self adjointness of elliptic operators with an infinite number of variables. Ukrainian. Math. J. 1975, 27, 729-742.

35. Gross, L. Potential theory on a Hilbert space. Jour. Funct. Ana. 1967, 1, 123-181.

36. Hida, T. Analysis of Brownian functionals; Carleton. Maths. Lect. Notes. 13, Carleton University Press: Carleton, Canada, 1975.

(c) 2009 by the author; licensee Molecular Diversity Preservation International, Basel, Switzerland. This article is an open-access article distributed under the terms and conditions of the Creative Commons Attribution license http://creativecommons.org/licenses/by/3.0/. 